\title{
Locating the contradiction in our understanding of time
}

\author{
Simon Prosser \\ University of St Andrews
}

Peer commentary on Christoph Hoerl and Teresa McCormack, 'Thinking in and About Time: A Dual Systems Perspective on Temporal Cognition' Behavioral and Brain Sciences

Hoerl and McCormack say that 'there is an inherent contradiction in people's naïve theory of time, in so far as it contains within it both the belief that there is an objective present and also the belief that which moment in time is objectively present changes.' (p. 49). They suggest that their two-system account helps explain this. But although I think there is something to be said for their proposal, the contradiction that they explain is different from the one that they say they are going to explain.

The latter contradiction arises because of the alleged conflict between the claim that the present time has a unique, privileged status and the claim that every time momentarily becomes present as time passes. It cannot be true that all times are present, and that only one time is present. As Hoerl and McCormack suggest, this is one way of capturing the contradiction that J. M. E. McTaggart (1908) suggested lay in the very notions of past, present and future.

There is a standard response: no contradiction arises in our naive view because, according to that view, only one time is present at any given time. Hoerl and McCormack say that this reply fails because 'it makes which moment in time is present dependent on what time it is considered from, rather than it being an objective property of time which moment is present.' (p. 50). But this reply would not satisfy the advocate of the naive view. For they hold that when time passes, reality, as a whole, changes; a different time becomes present. This change does not correspond merely to a difference in perspective; it is an objective change in reality. No two times are present within a single reality, so there is no contradiction.

The contradiction that Hoerl and McCormack subsequently explain does not, however, appear to be the one whose existence I have just denied. Instead, it is a contradiction between the claim that there is just one moment in time, with past and future times not being real, and the claim that all times are equally real. This does not concern presentness; it concerns ontology, or what exists. Their suggestion is that because the temporal updating system represents only the present time, and deals with changes just by updating its model of the present, it disposes us to think that the content of the model is all that is real. The temporal reasoning system, by contrast, represents the whole time series, and thus drives the intuition that all times are real. 
It is worth mentioning some relevant theories in metaphysics. According to presentism, reality is not extended in time, and consists only of the present, whose features change as time passes. So the temporal updating system would embody the presentist view of reality, and explains the intuition that drives it. According to eternalism, by contrast, reality is extended in time, and all times are equally real. The temporal reasoning system thus models an eternalist metaphysics.

Not all eternalists deny that time passes, however. Some, known as moving spotlight theorists, hold that each time undergoes constant changes in the extent to which it is past, present or future. The fact that such views are possible, and sometimes advocated, must cast some doubt on whether the two-systems theory can explain the sense of time passing, rather than explaining just the ontological intuition that only what is present is real (though this would still be progress).

Presentists do not deny that there are truths about the past or future. They typically hold that reality should be described using the operators of tense logic, such as in the past'. Thus the past occurrence of rain is represented as 'in the past: it is raining'. This corresponds to one version of our naive view of time: only the present is real, but it is nonetheless true to say that various things have happened, or will happen. Does the temporal reasoning system provide a representation that conflicts with this naive view? It is not clear that it must do so, in order to provide cognitive benefits relative to the temporal updating system. The temporal reasoning system represents times in an order, perhaps with a metric. But this would be true even if the representation were from the perspective of the present, with descriptions of events at other times preceded by the appropriate tense operators. Since this is compatible with presentism, it ought not to contradict the representation of the temporal updating system.

To produce the relevant ontological contradiction, then, what is needed is a representation of time as a dimension, an extended region of reality all of whose parts exist equally. Contrast this with, say, a system of representation of voltages and currents in an electrical circuit. When one plots a graph of voltage against current, one does not thereby draw a map of an extended region of reality. But when one plots a region of space along two axes, one does draw such a map. The difference lies not in the system of representation itself, which in both cases involves an ordering and a metric, but in the way in which this representation is interpreted.

Hoerl and McCormack do describe the temporal reasoning system as representing time as a dimension, but they do not say much about what makes this the case. Perhaps one important factor would be the ability to represent the time series from no specific point of view (and thus without representing times in terms of tenseproperties such as past; note the impossibility of a corresponding representation in the voltage/current example, with axes that do not represent properties). This raises the question of whether there could be an intermediate case in which a creature has a reasoning system that represents a series of times identified by their tenses, allowing cognitive advantages over a creature with only a temporal updating system, but without the decentered, 'no-perspective' representation that would contradict the presentism suggested by the temporal updating system. 


\section{References}

McTaggart, J.E. (1908). The unreality of time. Mind, 17(4), 457-474. doi:10.1093/mind/XVII.4.457 